\title{
Estudo comparativo in vitro entre biopróteses de pericárdio bovino e porcinas
}

\author{
Domingo M. BRAILE*, Moacir Fernandes de GODOY*, Dorotéia R. S. SOUZA*, Ana Paula M. L. \\ OLIVEIRA* ${ }^{*}$, João Carlos LEAL*, Renato B. ARAUJO*, Gilberto GOISSIS**
}

RBCCV $44205-319$
Braile D M, Godoy M F, Souza D R S, Oliveira A P M L, Leal J C, Araújo R B, Goissis G - Estudo comparativo in vitro entre biopróteses de pericárdio bovino e porcinas. Rev Bras Cir Cardiovasc 1996; 11 (4): 270-3.

RESUMO: A maioria dos implantes valvulares cardiacos realizados no Brasil é representada pelas válvulas de pericárdio bovino, seguidas por próteses porcinas. Na avaliação de válvulas biológicas, deve-se considerar: desempenho hidrodinâmico, resistência à fadiga e processo de calcificação. No presente estudo, foi avaliado o desempenho hidrodinâmico de biopróteses de pericárdio bovino (Biopro-PB-Braile Biomédica) comparativamente às válvulas porcinas (Biopro-PP-Braile Biomédica) através do gradiente médio transvalvular. Os testes hidrodinâmicos foram realizados em próteses de diâmetros variando de 19 a $35 \mathrm{~mm}$, submetidas ao Sistema Duplicador de Pulsos Shelhigh (Shelhigh Inc.). O volume de ejeção foi mantido constante em 90 $\mathrm{ml}$, com freqüência de pulso de $60,70,80,90$ e 100 ciclos por minuto, possibilitando fluxos entre 5 e 9 litros por minuto, equivalentes a fluxos contínuos aproximados de 8 a 18 litros por minuto. Houve tendência à diminuição dos gradientes pressóricos à medida em que aumenta o diâmetro externo das próteses. 0 gradiente pressórico médio encontrado em próteses de pericárdio bovino foi significativamente menor que 0 de próteses porcinas $(p<0,01)$, para todos os diâmetros estudados. Em vista da grande variabilidade dos protocolos de testes encontrados na literatura, reforça-se a necessidade de testes padronizados para avaliaçăo hidrodinâmica de próteses valvulares, visando comparaçāo adequada entre os diferentes modelos existentes.

DESCRITORES: Prótese das valvas cardiacas. Bioprótese. Pericárdio. Pericárdio, bovinos. Pericárdio, suinos. Teste de materiais. Materiais biocompativeis.

\section{INTRODUÇÃO}

O perfeito desempenho das valvas cardíacas depende da interação funcional entre parede atrial, cordoalha tendinosa, músculos papilares e parede ventricular. Doenças congênitas, reumáticas, infecciosas e isquêmicas podem prejudicar essa inte- ração, levando à necessidade de reparo valvar ou, eventualmente, sua substituição por prótese biológica ou mecânica. A melhora na qualidade e na duração da vida depende diretamente do desempenho hidrodinâmico do dispositivo empregado. Espera-se que ocorra baixa resistência ao fluxo sangüíneo

\footnotetext{
Trabalho realizado em Braile Biomédica, São José do Rio Preto, SP, e Instituto de Química de São Carlos - USP, São Carlos, SP, Brasil. Apresentado ao $23^{\circ}$ Congresso Nacional de Cirurgia Cardiaca. Recife, PE, 20 à 23 de março 1996.

* Da Divisão de Pesquisas e Publicaçōes - Braile Biomédica.

** Do Departamento de Quimica e Fisica Molecular, Grupo de Bioquímica - Instituto de Química de São Carlos - USP.

Este trabalho teve apoio do Conselho Nacional de Desenvolvimento Cientifico e Tecnológico (CNPq/PADCTII, 620. 628. 658/49) e Braile Biomédica, Indústria, Comércio e Representações S/A.

Endereço para correspondência: Domingo M. Braile. Av. Juscelino Kubitschek, 3101. São José do Rio Preto, SP, Brasil. CEP: 15091-450. Fax: [017] 227-3171.
} 
Braile D M, Godoy M F, Souza D R S, Oliveira A P M L, Leal J C, Araújo R B, Goissis G - Estudo comparativo in vitro entre bioprótese de pericárdio bovino e porcinas. Rev Bras Cir Cardiovasc 1996; 11 (4):270-3 .

e refluxo mínimo ou ausente durante o fechamento valvular.

Desde os trabalhos pioneiros de IONESCU et al. (1), diferentes tipos de próteses biológicas têm sido propostos. No Brasil, a maioria dos implantes valvulares cardiacos é representada pelas válvulas de pericárdio bovino, seguidas por próteses porcinas. O presente trabalho visa comparar o desempenho hidrodinâmico de próteses de pericárdio bovino e porcinas em termos de gradiente médio transvalvular.

\section{MATERIAL E MÉTODOS}

O estudo foi conduzido no Laboratório de Testes Hidrodinâmicos da Divisão de Pesquisas e Publicaçōes da Braile-Biomédica, com biopróteses de pericárdio bovino (Biopro-PB) e porcinas (Biopro-PP) da Braile Biomédica Indústria Comércio e Representações S.A. de São José do Rio Preto, São Paulo.

Foram utilizadas válvulas com diâmetros externos de $19,21,23,25,27,29,31,33$ e $35 \mathrm{~mm}$, submetidas a testes na posição mitral, em sistema duplicador de pulsos da Shelhigh Inc. (New Jersey, USA). Esse sistema incorpora dois amplificadores, fluxômetro eletromagnético, transdutor diferencial de pressão, computador PC AT286, impressora e sensor eletromagnético para medida de fluxo.

Avaliou-se, como parâmetro hidrodinâmico, o gradiente pressórico médio transvalvular em milimetros de mercúrio, empregando-se volume de ejeção fixo $(90 \mathrm{ml})$ e freqüências de pulso de 60,70 , 80,90 e 100 batimentos por minuto. Isto possibilitou fluxos entre 5 e 9 litros por minuto, equivalentes a aproximadamente 8 a 18 litros por minuto de fluxo contínuo (steady flow). As próteses foram submetidas a 5 testes por freqüência de pulso, totalizando 25 testes para cada diâmetro, ou seja, um conjunto de 450 testes.

A análise estatística compreendeu testes de análise de variância com nível de significância de $1 \%$.

\section{RESULTADOS}

Os gradientes mínimo, máximo e médio, e desvio padrão, com base nos 25 testes (fluxo contínuo de 8 a 18 litros/minutos) para cada um dos diâmetros estudados das biopróteses de pericárdio bovino e porcinas, encontram-se nas Tabelas 1 e 2, respectivamente, e no Gráfico 1. Nota-se nítida tendência à diminuição dos gradientes pressóricos, à medida em que aumenta o diâmetro externo das biopróteses. Os valores encontrados para as válvulas de pericárdio bovino foram hemodinamicamente mais
TABELA 1

GRADIENTES TRANSVALVULARES MINIMO, MÁXIMO, MÉDIO E DESVIO PADRĀO (EM $\mathrm{mmHg}$ ) DE BIOPRÓTESES DE PERICÁRDIO BOVINO COM DIÂMETROS EXTERNOS DE 19 A $35 \mathrm{~mm}$, SUBMETIDAS A TESTE HIDRODINÂMICO EM DUPLICADOR DE PULSO COM FLUXO CONTÍNUO (8,0 A 18,0 I/min)

\begin{tabular}{crrrr}
\hline \multirow{2}{*}{$\begin{array}{c}\text { PRÓTESE } \\
\text { (MM) }\end{array}$} & \multicolumn{3}{c}{ GRADIENTE } & \multirow{2}{*}{ DESVIO } \\
\cline { 2 - 3 } & MÍNIMO & MÁXIMO & MÉDIO & PADRÃO \\
\hline 19 & 13,0 & 21,0 & 18,2 & 2,4 \\
21 & 7,9 & 17,2 & 13,0 & 3,1 \\
23 & 5,2 & 13,4 & 9,3 & 2,7 \\
25 & 4,3 & 10,3 & 7,4 & 2,0 \\
27 & 4,2 & 9,6 & 7,0 & 1,8 \\
29 & 2,5 & 6,9 & 4,8 & 1,5 \\
31 & 3,8 & 7,7 & 5,2 & 1,2 \\
33 & 2,7 & 6,2 & 4,5 & 1,1 \\
35 & 2,1 & 6,0 & 3,8 & 1,3 \\
\hline
\end{tabular}

TABELA 2

GRADIENTES TRANSVALVULARES MINNIMO, MÁXIMO, MÉDIO E DESVIO PADRĀO (EM $\mathrm{mmHg}$ ) DE BIOPRÓTESES PORCINAS COM DIÂMETROS EXTERNOS DE 19 A $35 \mathrm{~mm}$, SUBMETIDAS A TESTE HIDRODINÂMICO EM DUPLICADOR DE PULSO COM FLUXO CONTINNUO $(8,0$ A 18,0 L/min)

\begin{tabular}{ccccc}
\hline \multirow{2}{*}{$\begin{array}{c}\text { PRÓTESE } \\
\text { (mm) }\end{array}$} & \multicolumn{3}{c}{ GRADIENTE } & \multirow{2}{*}{ DESVIO } \\
\cline { 2 - 3 } & MÍNIMO & MÁXIMO & MÉDIO & PADRÃO \\
\hline 19 & 15,9 & 22,9 & 20,3 & 1,7 \\
21 & 16,5 & 22,9 & 20,4 & 1,7 \\
23 & 9,1 & 14,8 & 12,7 & 1,3 \\
25 & 7,8 & 17,3 & 13,2 & 3,3 \\
27 & 7,7 & 17,9 & 12,8 & 3,2 \\
29 & 5,6 & 12,6 & 9,1 & 2,3 \\
31 & 4,5 & 8,6 & 6,3 & 1,4 \\
33 & 4,3 & 8,9 & 6,3 & 1,4 \\
35 & 3,2 & 6,9 & 4,8 & 1,2 \\
\hline
\end{tabular}

GRÁFICO 1

GRADIENTES MÉDIOS TRANSVALVULARES DE BIOPRÓTESES DE PERICÁRDIO BOVINO E PORCINAS SOB FLUXO CONTINUO (8,O A 18,O L/MIN), DE ACORDO COM O DIÂMETRO EXTERNO.

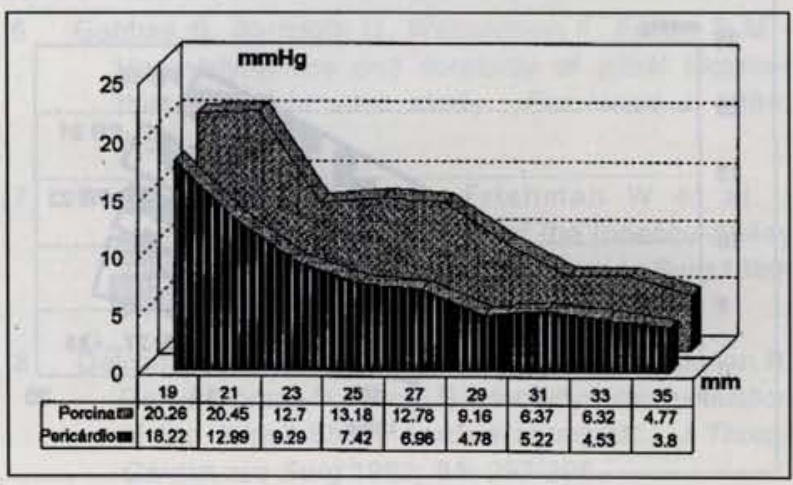


Braile D M, Godoy M F, Souza D R S, Oliveira A P M L, Leal J C, Araújo R B, Goissis G - Estudo comparativo in vitro entre bioprótese de pericárdio bovino e porcinas.

Rev Bras Cir Cardiovasc 1996; 11 (4):270-3

favoráveis que os obtidos para as porcinas em fluxos equivalentes. A diferença entre os gradientes médios das biopróteses de pericárdio bovino e porcinas foi estatisticamente significativa $(p<0,01)$ para todos os diâmetros externos estudados.

Houve aumento progressivo do gradiente pressórico em relação direta com o aumento do fluxo transvalvular. Os gradientes transvalvulares nas biopróteses de pericárdio bovino agruparam-se em 4 faixas bem definidas (válvulas de $19 \mathrm{~mm}, 21 \mathrm{~mm}, 23 \mathrm{~mm}$ e de 25 a $35 \mathrm{~mm}$ ), enquanto que as porcinas mantiveram-se agrupadas em 3 faixas (válvulas de 19 e 21 $\mathrm{mm}, 23$ a $29 \mathrm{~mm}$ e de 31 a $35 \mathrm{~mm}$; Gráficos 2 e 3).

\section{COMENTÁRIOS}

Os estudos clássicos e básicos a respeito da avaliação hidrodinâmica de próteses valvulares foram realizados por GABBAY et al (2), no final da década de 70. A partir de então, uma série de trabalhos têm aparecido na literatura, utilizando duplicadores de pulso para avaliar o desempenho de próteses mecânicas $(3,4)$ e biológicas com ou sem suporte ${ }^{(5)}$. A comparação entre os vários trabalhos, porém, é bastante difícil, pois a diversidade de métodos empregados tem tornado praticamente impossivel a extrapolaçāo dos resultados para aplicação geral. Encontram-se relatos cujos testes foram realizados não individualmente por diâmetro, mas por grupos de tamanho: pequenas, médias e grandes (6). Outros autores avaliaram o desempenho da bioprótese por meio do gradiente transvalvular in vivo, logo ao final da circulação extracorpórea (7), ou então no pós-operatório tardio por cateterismo cardíaco (8). É claro que esses resultados não podem

GRÁFICO 2

GRADIENTES TRANSVALVULARES MEDIOS DE BIOPRÓTESES DE PERICARDIO BOVINO COM DIÁMETROS DE 19, 21, 23, 25, 27, 29, 31, 33 E 35 MM, DE ACORDO COM O FLUXO (STEADY FLOW), NOTANDO-SE TENDÊNCIA DE AGRUPAMENTO EM 4 FAIXASDEFINIDAS.

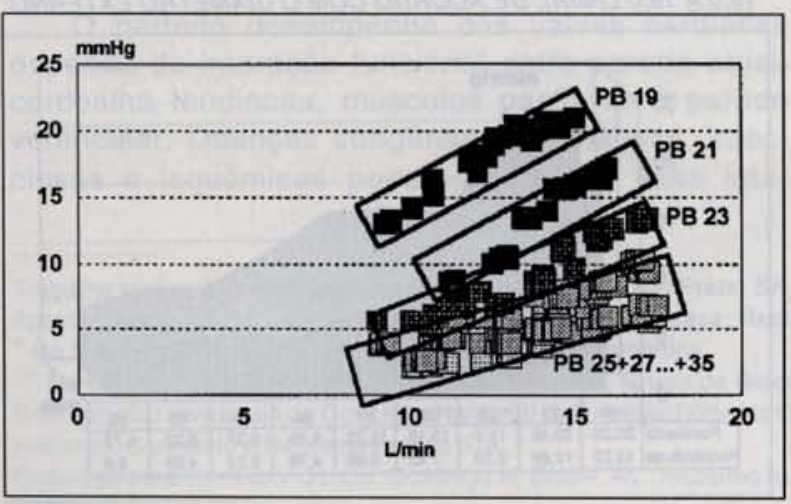

ser comparados a outros em que os testes foram individualizados e/ou realizados em duplicadores de pulso.

Além disso, nota-se quase que completa aleatoriedade em termos do fluxo transvalvular utilizado, sendo empregadas diversas combinações de volume de ejeção e freqüência (5). É sabido que um mesmo fluxo pode ser obtido por combinações variáveis de freqüência e stroke. Não se pode garantir, a menos que testadas todas as possibilidades, que as condições sejam as mesmas nos vários experimentos publicados. A própria constituição do meio hidrodinâmico (composição, viscosidade etc), em que a prótese é colocada, pode interferir nos resultados.

Finalmente, o número de experimentos ao qual cada prótese é submetida varia muito. Quanto mais testes foram realizados com uma única prótese, em termos de variaçōes de freqüência de pulso ou fluxo transvalvular, o resultado obtido será certamente a tradução mais próxima da realidade hidrodinâmica. Alterações no sistema de testes podem ser responsáveis por modificações que estejam sendo implantadas ao funcionamento da prótese.

Assim, torna-se imperiosa a rigorosa padronização de testes de avaliação hidrodinâmica das próteses. Dispondo do sistema padrão de testes hidrodinâmicos de próteses valvulares (Shelhigh Inc.), têm sido testadas, sistematicamente, neste Laboratório, biopróteses de pericárdio bovino e porcinas, visando determinar os valores hidrodinâmicos normais para esses tipos de válvulas. O equipamento permite a aferição dos seguintes parâmetros: gradiente médio transvalvular, gradiente no fluxo máximo, volume de fechamento, volume de vazamento, fração regurgitante, área orificial efetiva, coeficiente de descarga, indice de desempenho e ín-

GRÁFICO 3

GRADIENTES TRANSVALVULARES MÉDIOS DE BIOPRÓTESES PORCINAS COM DIAMMETROS DE 19, 21, 23, 25, 27, 29, 31, 33 E $35 \mathrm{MM}, D E$ ACORDO COM O FLUXO (STEADY FLOW), NOTANDO SE TENDÉNCIA DE AGRUPAMENTO EM 3 FAIXAS DEFINIDAS.

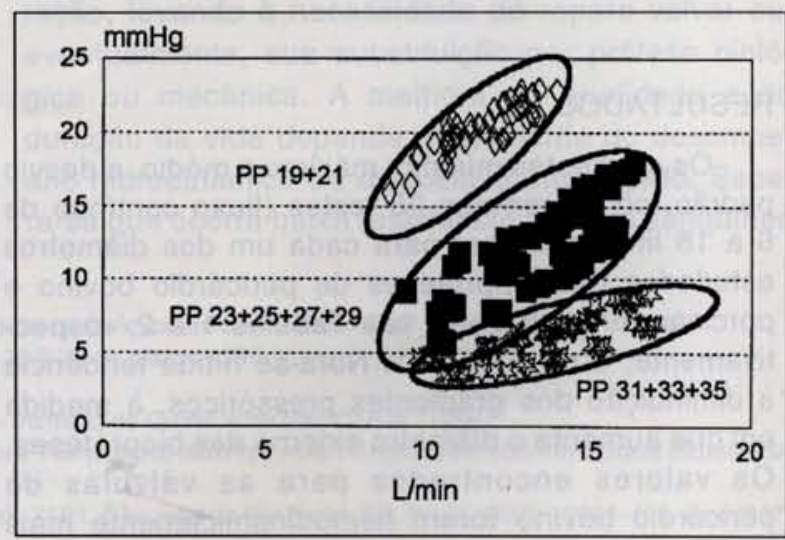


Braile D M, Godoy M F, Souza D R S, Oliveira A P M L, Leal J C, Araújo R B, Goissis G - Estudo comparativo in vitro entre bioprótese de pericárdio bovino e porcinas. Rev Bras Cir Cardiovasc 1996; 11 (4):270-3 .

dice de eficiência. A padronização rigorosa em termos das condições do sistema e da regulação do volume de ejeção e freqüência, além do número suficiente de testes (25) de cada prótese, permitirá a adequada comparação dos resultados.

No presente estudo foram medidos e comparados os resultados hidrodinâmicos de biopróteses de pericárdio bovino e porcinas, em termos de gradiente médio transvalvular sob fluxo contínuo (steady flow). Verificou-se diferença estatisticamente significativa entre as biopróteses de pericárdio bovino e as porcinas $(p<0,01)$ para todos os fluxos estudados, com vantagem para as próteses de pericárdio. O melhor desempenho das biopróteses de pericárdio sobre as porcinas também tem sido verificado na literatura apesar das diferentes manufaturas $(2,5)$.
A comparação entre os resultados ora apresentados e os citados na literatura, para outros tipos de próteses, não teria rigor científico, pois, como já dito, os protocolos utilizados são diferentes nos diversos trabalhos consultados. Além disso, a grande maioria das próteses estudadas já se encontra fora de uso clínico, ou sofreu modificações após aquelas publicações, o que torna a comparação sem utilidade prática.

Assim sendo, além de apresentar o desempenho, em termos de gradiente transvalvular, das próteses biológicas utilizadas em nosso Serviço, este trabalho tem o objetivo de propor a criação de um consenso, pelo menos a nível nacional, para normatização e padronização de testes hidrodinâmicos, visando à perfeita comparabilidade dos diversos tipos de prótese existentes em nosso meio.

RBCCV $44205-319$

Braile D M, Godoy M F, Souza D R S, Oliveira A P M L, Leal J C, Araújo R B, Goissis G - In vitro comparative study between bovine pericardial and porcine bioprosthesis. Rev Bras Cir Cardiovasc 1996; 11 (4): 270-3.

ABSTRACT: Most of the cardiac valve implantations in Brazil are represented by bovine pericardial valves, followed by the porcine prostheses. In the evaluation of biological valves, the following should be taken into consideration: hydrodynamic performance resistance to fatigue and calcification process. In this study, the hydrodynamic performance of bovine pericardial bioprostheses (Biopro-BP-Braile Biomedica) was evaluated comparatively to porcine valves (Biopro-PP-Braile Biomedica), through the transvalvular medium gradient. The hydrodynamic tests were made on prostheses varying from 19 to $35 \mathrm{~mm}$ in diameter, which underwent the pulse duplicator system Shelhigh (Shelligh Inc.). The ejection volume was constantly kept at $90 \mathrm{ml}$. The pulse frequencies varied between 60 and 100 cycles per minute, allowing flows between 5 and 9 liters per minute, equivalent to approximate continuos flows of 8 to 18 liters per minute. There was tendency to decrease the gradients, in proportion to increase of the bioprostheses external diameters. The medium pressure gradient found in tbe bovine pericardial prostheses was significantly smaller than that in the porcine prostheses $(p<0.01)$, for all diameters studied. Respecting the large variability of protocols of tests in the literature for hydrodynamic evaluation of valve prostheses, the necessity of standard tests becomes stronger, seeking adequate comparison among the several valves.

DESCRIPTORS: Heart valves prosthesis. Bioprosthesis. Pericardium. Pericardium, cattle. Pericardium, swine. Material testing. Biocompatible materials.

\section{REFERÊNCIAS BIBLIOGRÁFICAS}

1 Ionescu M I, Pakrashi B C, Mary D A S, Bartek I T, Wooler G H - Long-term evaluation of tissue valves. $J$ Thorac Cardiovasc Surg 1974; 68: 361-79.

2 Gabbay S, McQueen D M, Yellin E L, Frater R W M. In vitro hydrodynamic comparison of mitral valve prostheses at high flow rates. JThorac Cardiovasc Surg 1978; 76: 771-87.

3 Okude J, Sakai K, Matsumani O, Tanabe T - In vitro evaluation of hydrodinamics of prosthetic heart valves. Life Support Systems 1986; 4(Supl.2): 33-5.

4 Umezu M, Nugent A, Ye C X, Chang V P. Hydrodynamic characteristics of newly-developed prosthetic heart valves by the use of several types of in vitro tests systems Jpn J Artif Organs 1989; 18: 719-22.
5 Walker D K, Scotten L N, Brownlee R T - New generation tissue valves. J Thorac Cardiovasc Surg 1984; 88: 573-82.

6 Gabbay S, Bortolotti U, Wasserman F, Factor S M Haemodynamics and durability of mitral bioprostheses: an in vitro study. Eur Heart $J$ 1984; 5(Supl.1): 65-71.

7 Becker R M, Strom J, Frishman W et al. Hemodynamic performance of the Ionescu-Shiley valve prosthesis. J Thorac Cardiovasc Surg 1980; 80: $613-20$.

8 Delcan J L, Chaitman B R, Lopez-Bescos L, Bonan R, Garcia-Dorado D, Rivera R - Hemodynamic evaluation of the Angell-Shiley porcine xenograft. $J$ Thorac Cardiovasc Surg 1982; 84: 297-305. 